\title{
Una experiencia de formación inicial de docentes de inglés para la inclusión de las TIC en la enseñanza de lenguas
}

\author{
An Experience of Initial English Teaching Training \\ Incorporating ICT in Language Teaching
}

\author{
Amparo Clavijo Olarte, Ph.D. ${ }^{1}$ \\ Luz Mary Quintero, M.A. ${ }^{2}$
}

\section{Resumen}

Este artículo muestra los hallazgos de un estudio realizado con un grupo de 34 estudiantes, de un programa de licenciatura en inglés como lengua extranjera de una universidad pública de Bogotá, que se enfoca en la necesidad de repensar la pedagogía del lenguaje con el fin de promover el desarrollo de competencias en lengua extranjera y en alfabetización digital en los estudiantes. El estudio analiza la pedagogía utilizada en el ambiente virtual basada en: 1. proyectos colaborativos, 2. debates sobre temas generados por los estudiantes, 3. diseño y construcción de blogs personales y 4. creación de wikis en el espacio de Schoolpedia. Los resultados muestran que la pedagogía propuesta, basada en proyectos colaborativos desarrollados por los estudiantes, representó una experiencia de aprendizaje del idioma enriquecida con la inclusión de TIC. De esta forma, podría convertirse en un modelo para ellos como futuros docentes. Esta experiencia pedagógica mostró en el currículo las relaciones de complementariedad entre los saberes lingüístico y tecnológico para la comunicación, y entre los contenidos escolares y los contenidos propios del estudiante en el aprendizaje.

\section{Palabras clave:}

Formación de docentes, inclusión de las TIC, pedagogía por proyectos.

\section{Abstract}

This paper presents the results of a study developed with a group of 34 TESOL students of a public university at Bogotá, Colombia. The study focuses on the need to rethink the pedagogy of language in order to promote the development of foreign language competencies and digital literacy among students. The study analyzes the pedagogy implemented in virtual environments, which is based on: 1. collaborative projects, 2. debates on topics proposed by students, 3 . design and development of personal blogs, and 4. creations of wikis in Schoolpedia. The results show that the pedagogy based on collaborative projects developed by students turned out to be an enriched learning experience with the inclusion of ICT; thus, this pedagogy could become a model for TESOL students future teaching practice. The inclusion of this pedagogical experience into the curriculum also showed complementary relationships between teaching contents and students' personal interests, which have consequences to learning.

Keywords:

Teaching training, ICT inclusion, project pedagogy.

Artículo recibido el 26 de abril y aprobado el 19 de julio de 2012

1 Universidad Distrital Francisco José de Caldas. Maestría en Lingüística Aplicada a la Enseñanza del Inglés, Bogotá, Colombia. Correo electrónico: aclavijoolarte@yahoo.com, maestria@udistrital.edu.co

2 Universidad Industrial de Santander. Bucaramanga, Colombia. Correo electrónico: luzmaq03@yahoo.com 
El creciente uso de las TIC por parte de los estudiantes de todos los niveles educativos, fuera del ambiente escolar, representa un reto para los docentes en ejercicio y, por lo tanto, para los profesores en formación, en tanto han tenido que repensar una pedagogía que integre exitosamente las TIC en el currículo escolar. Así, tanto los profesores en ejercicio como los que están en formación deben prepararse para asumir la educación de los estudiantes de este tiempo, quienes han crecido utilizando diferentes tipos de tecnologías y esperarían encontrarlas en actividades educativas en la escuela.

Este artículo tiene como propósito presentar algunos aspectos centrales de la pedagogía utilizada para el trabajo en una plataforma virtual (www.ourdigitalculture.com), con un grupo de 34 estudiantes de un programa de licenciatura en educación básica con énfasis en inglés como lengua extranjera, de una universidad pública de Bogotá. La pedagogía se describe en torno a cuatro principios: participación, colaboración, desarrollo de proyectos pedagógicos y personalización del espacio virtual, comunicación. El trabajo en la plataforma se desarrolló en tres espacios: blog personal, blog de debates y un espacio de construcción colectiva similar a un wiki. Dada la naturaleza de la herramienta, el trabajo de los estudiantes, en su mayoría, se evidenció a través de la escritura en los espacios anteriormente mencionados, y en algunas ocasiones en videoconferencias que no serán discutidas para los propósitos del presente artículo. El trabajo se desarrolló en la plataforma www.ourdigitalculture.org dispuesta por la Universidad de Dundee, en Escocia. En la plataforma estaban vinculados estudiantes de diferentes nacionalidades que interactuaban para compartir la historia, literatura y la cultura de los diferentes países, a la vez que usaban significativamente el idioma que estaban aprendiendo, en nuestro caso el inglés. Las actividades en la plataforma virtual fueron construidas con objetivos estrictamente pedagógicos, de manera que se iba ajustando según las necesidades e intereses de los docentes y estudiantes participantes.

Nuestra propuesta pedagógica se fundamenta teóricamente en la perspectiva social y cultural del lenguaje propuesta por Vygotsky (1978), Dewey (1938) y Freire y Macedo (1987), cuyos fundamentos filosóficos han contribuido a pensar los procesos de enseñanza y aprendizaje a partir de la construcción de experiencias significativas para los aprendices, a través de la indagación y la solución de problemas sociales en su entorno, y como una forma de participación y construcción colaborativa entre pares.

Desde esta perspectiva Vygotsky (1978), Moll (1990) y Wells (1999) consideran los procesos de lectura y escritura como prácticas sociales y herramientas culturales que permiten diversas formas de representación y comunicación a los individuos, como seres sociales. Estas diversas formas de representación del mundo a través de la lectura y la escritura, como prácticas sociales y culturales, han cambiado a través de los años, de acuerdo al acceso a la cultura escrita y a la participación de los individuos de una sociedad (Kalman, 2003) en la comunicación con otros. Actualmente, las nuevas prácticas sociales de lectura y escritura mediadas por las tecnologías de la información y la comunicación (TIC), la conectividad que permite el acceso a la información en Internet y a la formación de mundos y comunidades virtuales, el acceso libre al software social que ofrece la Web 2.0 (New Media Consortium, 2005), nos muestran que existen nuevas formas de interacción para la comunicación con otros, que requieren nuevos paradigmas pedagógicos para incorporarlos en la escuela exitosamente.

Clavijo, Quintana y Quintero (2011) consideran que estos nuevos medios dan lugar al surgimiento de un nuevo ecosistema mediático cultural, en el cual los sujetos tienen nuevas necesidades de formación para una actuación más participativa, autónoma y en relación de pares que acentúe la horizontalidad de las interacciones. A este respecto Igarza, en Clavijo, Quintana y Quintero (2011) ilustra lo siguiente:

Los nuevos medios son nuevas formas culturales que dependen de una computadora para su distribución, representación y uso interactivo... son formas culturales que dependen de componentes digitales físicos para ser almacenadas, distribuidas y representadas... los nuevos medios explotan las capacidades del procesador para ofrecer 
una experiencia interactiva y la conectividad a la red para el acceso remoto a los contenidos y la comunicación entre usuarios... son sistemas hipermedia adaptativos que proponen una experiencia de inmersión en un entorno permeable, crecientemente inteligente y personalizable, que tiende a involucrar al usuario en una sucesión de toma de decisiones potencialmente sin fin. (Igarza, 2008, p. 11)

Estas nuevas formas culturales de comunicación y de aprendizaje, según Clavijo y otros (2011), implican ver, desde una perspectiva crítica, el rol de la escuela y la universidad en la formación de las nuevas generaciones de niños y niñas, de manera que "ellos puedan disponer, en el futuro cercano, de los conocimientos y habilidades que les permitan ser partícipes de la realidad, altamente tecnológica, en la que viven, desarrollen competencias en el uso de la alfabetización digital y la alfabetización mediática, pero también la alfabetización ciudadana, y la económica" (Dussel y Southwell, 2007, p. 2, citado en Clavijo y otros, 2011). Además, para que los niños y niñas tengan la capacidad de aplicar conceptos científicos y tecnológicos a la vida, el trabajo y la cultura propia de la sociedad o contexto donde se desenvuelvan, denominado por la Unesco como alfabetización científica y tecnológica (p. 24).

Estas nuevas formas culturales de comunicación entre aprendices que usan las nuevas tecnologías, a su vez requieren ver la enseñanza del inglés desde otro paradigma diferente al tradicional de la escuela, en donde el idioma extranjero es una asignatura de un currículo fijo, que se desarrolla en un aula de clase como el único espacio para aprender y, en cambio, concebirla desde una perspectiva pedagógica donde la participación, la colaboración y la comunicación sean elementos básicos para comprender la lengua extranjera en contextos más reales de uso. Así, creemos que el aprendizaje mediado por herramientas tecnológicas y herramientas de la Web 2.0 facilita, tanto al docente como al estudiante, la comunicación con otros usuarios de la lengua, nativos y no nativos, a través de proyectos pedagógicos.

El uso de estos ambientes de aprendizaje para la participación, la colaboración y la comunicación, por supuesto, imponen en los docentes múltiples requisitos y retos. Entre otros, la necesidad de desarrollar competencias de alfabetización digital, de medios, científica y tecnológica para poder usar las TIC en la enseñanza; la necesidad de adoptar un papel propositivo de situaciones de aprendizaje y de participación innovadoras y la necesidad de estar permanentemente en comunicación en red con otros docentes y estudiantes; e indiscutiblemente, la necesidad de trabajar colaborativamente con otros docentes e incluso con estudiantes. Estas nuevas realidades para la escuela y la sociedad, a su vez, requieren de decisiones, acciones y políticas institucionales por parte de las instituciones de educación superior, para que los programas de formación inicial y formación avanzada de docentes ofrezcan experiencias enriquecidas con las TIC que apoyen a los docentes en estos desarrollos.

En cuanto a política pública sobre educación, Colombia, como muchos otros países, promueve la integración de las TIC en la educación en todos los niveles. El programa Plan TIC Colombia 2008-2019 plantea el desarrollo de un sistema educativo incluyente, que ofrezca oportunidades de aprendizaje autónomo y colaborativo mediado por las TIC. Para lograr los objetivos, el plan propone acciones específicas en tres áreas estratégicas: gestión de la infraestructura, gestión de contenidos y gestión del recurso humano, entendido como maestros y estudiantes. El país ha tomado acciones en estas tres áreas estratégicas desde el Ministerio de las Tecnologías de la Información y las Comunicaciones (www.mintic.gov.co), las Secretarías de Educación (www.redacademica.edu.co) y el portal educativo Eduteka, con el fin de alcanzar las metas propuestas y hacer posible el uso de las TIC en la escuela. El interés del presente artículo se centra en el tercer factor, considerado estratégico: gestión de contenidos y gestión del recurso humano, maestros y estudiantes, especialmente en el recurso humano-maestros. Para tal propósito discutiremos brevemente la importancia de la formación en TIC de los futuros maestros, con especial énfasis en los de inglés como lengua extranjera.

Zhao y Bryant (2007) afirman que para que los docentes integren efectivamente la tecnología 
en el currículo de la escuela, es necesario que se formen a partir de un currículo que les ofrezca una riqueza y variedad de usos pedagógicos de las herramientas tecnológicas, de manera que estas mismas experiencias de su formación, sirvan de modelos para que más adelante integren la tecnología en el currículo de la escuela. Los mismos autores consideran que la formación de los docentes en tecnología que solo se enfoca en la enseñanza de habilidades básicas del uso del computador, no asegura que ellos puedan incluirla en el aula de clase. Innegablemente, todos los programas de formación de docentes deben incluir asignaturas específicas dedicadas a enseñarle a los futuros docentes el uso básico de herramientas tecnológicas y de la Web 2.0, así como del uso del computador; sin embargo, este es solo uno de los componentes de formación, sin dejar de lado la formación pedagógica. Al respecto, Jacobsen, Clifford y Friesen (2002) señalan que las nuevas generaciones de profesores no pueden ser preparadas de la misma forma en que sus profesores fueron formados, porque los futuros docentes tendrán que enseñar a niños y adolescentes que pertenecen a una nueva generación, que ha nacido y crecido con la tecnología. Así, los profesores en formación deben encontrar buenos modelos de inclusión efectiva de las TIC a lo largo de su programa de formación en la universidad.

Visto de esta manera, los futuros profesores de inglés de nuestro país necesitan tener conocimiento y destrezas en el manejo de las tecnologías y a su vez, conocimiento pedagógico que les permita incorporarlas efectivamente en el currículo de lengua extranjera. Mishra y Khoeler (2006) proponen un modelo de formación en TIC denominado TPCK (Tecnological Pedagogical Content Knowledge), que en español significaría conocimiento del contenido tecnológico y pedagógico, definido por los autores como el conocimiento de la existencia, componentes y usos de las diferentes tecnologías usadas en los contextos de enseñanza y aprendizaje, y a la vez, el conocimiento y la comprensión de cómo la enseñanza y el aprendizaje pueden transformarse como resultado del uso apropiado de tecnologías particulares que se ajusten a los propósitos educativos.
En conclusión, los profesores necesitan no solo el conocimiento disciplinar (enseñanza del inglés y de la lengua), sino también conocimiento de la manera como la enseñanza de los contenidos disciplinares puede transformarse como resultado del uso efectivo de la tecnología. El estudio que presentamos intentó convertirse en un modelo de uso efectivo de las TIC en la enseñanza de una asignatura de inglés; de ninguna manera incluye todos los elementos descritos arriba por Mishra y Khoeler (2006), pero sí contribuye a despertar conciencia sobre el rol de la tecnología en los futuros docentes. Por lo tanto, se pensó tanto en el tipo de herramientas tecnológicas que se usarían, como en el tipo de pedagogía que fundamentaría el desarrollo del proyecto. A continuación describimos el tipo de pedagogía utilizada.

\section{Pedagogía de la participación y la colaboración a través de proyectos colaborativos}

La pedagogía de la participación y la colaboración que utilizamos en este estudio con el grupo de docentes en formación inicial, está orientada a promover en los estudiantes formas auténticas de lectura y escritura en inglés como lengua extranjera, mediante procesos de indagación sobre temas de interés social y cultural de los estudiantes que fueron plasmados luego en múltiples textos.

Esta pedagogía de la participación y la colaboración, se fundamenta teóricamente en los principios filosóficos y pedagógicos de la pedagogía de la liberación de Freire y Macedo (1987), centrada en la participación activa de los aprendices a través de la generación de preguntas para la indagación; así como en las ideas y principios progresistas de Dewey (1939), quien también nos muestra el camino de la indagación como forma posible para la construcción colectiva de saberes, lo que aquí también llamamos proyectos pedagógicos colaborativos. Short (2001), a su vez, desde su perspectiva como educadora, considera que la pedagogía de la indagación nos permite ofrecer un proceso de aprendizaje significativo, en donde se exploren diferentes lenguajes y modalidades de aprendizaje desde las diferentes disciplinas del saber. 
El aprendizaje a través de la indagación, la generación de preguntas, la exploración y la autoría de textos puede ser posible ahora mediante el uso de herramientas de las tecnologías de la información y la comunicación, disponibles en la Web 2.0. Estas herramientas, que están al alcance de docentes y aprendices, han sido denominadas por McLoughlin y Lee (2008) como el "software social", caracterizado por "hacer posible una nueva forma de colaboración distribuida e interacción social con un efecto transformador en la sociedad y en particular en la educación" (p. 12).

Siemens (2007) considera que la Web 2.0 ofrece nuevas alternativas a la educación, gracias a su estructura participativa y colaborativa que permite a los jóvenes, hoy día, participar y aprender conectados con múltiples mundos sociales virtuales. Este tipo de interacciones mediadas por las TIC difieren en gran medida de las interacciones que ocurren en las aulas de clase de las instituciones educativas y de los modos de aprendizaje, socialización y comunicación que tienen lugar en el día a día de la escuela. Estas interacciones y usos de las herramientas de la Web 2.0 para el aprendizaje dieron origen a lo que McLoughlin y Lee (2008) llaman la Pedagogía 2.0.

Los elementos centrales de la Pedagogía 2.0, según los autores, son la personalización, la participación y la productividad de contenidos. La personalización le brinda a los aprendices autonomía, agency, y autoregulación en su aprendizaje; la participación, por su parte, promueve la comunicación, la colaboración, la conectividad y la construcción de una comunidad; finalmente, la productividad permite a los estudiantes crear su propio contenido para el aprendizaje, ser generadores de conocimiento, dando así espacio para la creatividad y la innovación. (p. 16).

Una pedagogía de la participación y la colaboración mediada por las TIC se materializa en la construcción de proyectos pedagógicos colaborativos. Jolibert (1994) define los proyectos pedagógicos de aula como una de las estrategias para la formación de personas, que apunta a la eficiencia y eficacia de los aprendizajes y a la vivencia de valores democráticos, a través de un trabajo cooperativo, de co-elaboración del plan, de co-realización y de co-teorización que involucra a todos los actores: maestros y alumnos.

En la enseñanza de lenguas extranjeras, los proyectos colaborativos, además de los beneficios anteriormente mencionados, facilitan el desarrollo de la lengua, ya que estimulan y retan a los aprendices a usarla con propósitos auténticos. Stoller (2001) resalta que el aprendizaje de la lengua basado en proyectos, usualmente trae beneficios múltiples a los aprendices, tales como la construcción positiva del autoestima, confianza en y valoración de su saber, aprendizaje sobre temáticas diferentes a las relacionadas con el idioma y, por supuesto, avances significativos en el desarrollo de las habilidades de la lengua extranjera. Los proyectos colaborativos por naturaleza integran todas las habilidades (lectura, escritura, escucha y habla) y pueden ser usados con todo tipo de grupos, sin importar el nivel de proficiencia en la lengua.

\section{Método}

Los resultados reportados en el presente artículo hacen parte de un proyecto de investigación acción más amplio, el cual fue desarrollado por las autoras del artículo. El trabajo de aula se enfocó en observar, detalladamente, el proceso de apropiación y uso de las herramientas tecnológicas y su contribución en el aprendizaje de inglés como lengua extranjera. El rol de investigadores-docentes facilitó la interacción y la observación constante de los individuos, con el fin de entender la aprehensión y el uso de las herramientas digitales y de la lengua extranjera. Nuestro interés fue observar cómo los estudiantes desarrollaron competencias como usuarios de las TIC y del inglés como lengua extranjera, en la comunicación que tuvieron para compartir y construir colaborativamente conocimiento en el espacio de aprendizaje virtual. 


\section{Instrumentos para la recolección de datos}

\section{Blogs}

Los blogs representaron la más valiosa fuente de información para la investigación, ya que fueron los espacios creados por los estudiantes, y permitieron a los investigadores examinar las transformaciones que los estudiantes hicieron a sus textos a lo largo de la intervención pedagógica, a través del dispositivo version tracks, disponible en el espacio virtual. Adicionalmente, en los blogs se hizo evidente la interacción entre los estudiantes de instituciones educativas de distintos países, del mismo país o, incluso, entre estudiantes de la misma institución.

\section{Entrevistas}

Se hicieron entrevistas semiestructuradas, tanto a docentes como a estudiantes, al final de la intervención pedagógica. El propósito de la entrevista fue indagar sobre las opiniones que los participantes (maestros y estudiantes) en el proyecto de investigación tenían con respecto a las actividades pedagógicas desarrolladas, el funcionamiento de la plataforma virtual, la interacción con estudiantes de otros países o del mismo país y el impacto que tuvo la implementación del uso del espacio virtual y demás herramientas digitales y tecnológicas en la enseñanza y el aprendizaje, tanto para los estudiantes como para los docentes. En el momento de realizar las entrevistas, los participantes contaban con un nivel básico de inglés; por esta razón, decidimos hacer la entrevista en español para que así pudieran expresar sus ideas sin sentirse limitados por el idioma.

\section{Planificación y contexto de los proyectos colaborativos}

Este trabajo se desarrolló con los estudiantes del curso de inglés básico de segundo y tercer semestres, del programa de Licenciatura en Educación Básica con Énfasis en Inglés. El curso tenía una asignación de diez horas semanales, de las cuales se distribuyeron siete para desarrollar el programa del curso de inglés básico, siguiendo el texto asignado, y tres horas para llevar a cabo actividades en el ambiente virtual en un aula de informática. Las tres horas se distribuían así: una hora para participación en los debates, media hora para trabajar en el blog personal y el resto para trabajar en los wikis o universitypedia.

\section{Objetivos de enseñanza}

Los objetivos pedagógicos que orientaron el trabajo de los dos grupos de la universidad en el espacio virtual www.ourdigitalculture.org fueron:

1. Implementar actividades pedagógicas que promuevan el uso del idioma inglés con fines auténticos de comunicación, mediado por las TIC.

2. Estimular en los estudiantes el uso de la escritura como una forma de expresar, exponer y argumentar sus ideas.

3. Ofrecer un espacio virtual en el que los estudiantes puedan compartir y expresar libremente sus gustos e intereses con otras personas del salón de clase y de otros países.

4. Debatir temas actuales del interés de los estudiantes, con el fin de promover la escritura argumentativa en la lengua extranjera.

5. Explorar la cultura local a través de proyectos colaborativos para ser compartidos con estudiantes de otras culturas y para que, a su vez, los estudiantes redescubran y valoren su propia ciudad.

\section{Los proyectos colaborativos de estudiantes}

El trabajo en los espacios colaborativos se hizo en grupos generalmente de tres personas, donde cada una contribuía según sus habilidades. Así, los estudiantes debían visitar lugares de su comunidad, tomar fotos, encontrar información, redactar los textos y organizar todo en el espacio virtual acompañado de imágenes, música, animaciones, etc. Todos los textos que hicieron parte de los espacios colaborativos debían ser revisados por la docente una semana antes de ser publicados. Los textos grupales se preparaban generalmente fuera de clase, y el tiempo de clase lo dedicaban a organizar toda la información, la estética del espacio, a encontrar música, crear los hipervínculos y demás tareas que demandaba tener su trabajo publicado. 
El primer grupo desarrolló dos trabajos, uno sobre música colombiana y otro sobre lugares y eventos culturales y representativos de la ciudad de Bogotá. Para el primero, los estudiantes se dividieron en grupos de tres, le dieron un nombre a su equipo y crearon su propio grupo en el espacio de la plataforma dedicado al trabajo colaborativo. Durante tres meses los estudiantes trabajaron en equipo y escribieron sobre un género específico de música colombiana (pasillo, cumbia, joropo, porro, vallenato, carranga, etc.). Cada grupo escribió seis textos como resultado de la indagación. Cada espacio estaba conformado por una reseña sobre el tipo de música, fotografías o animaciones alusivas al tema y varias canciones sobre el género en formato MP3, para que los lectores pudieran deleitarse oyendo el género y así tener una mejor idea del tipo de música.

El segundo proyecto, lugares y eventos culturales y representativos de Bogotá, se desarrolló de forma similar. En tres meses los estudiantes escribieron sobre museos, iglesias, sitios turísticos, parques, bibliotecas, los humedales, el Festival de Teatro de Bogotá, Rock al Parque, Jazz al Parque, entre otros. $\mathrm{Al}$ igual que en el anterior proyecto, los textos resultado del trabajo, fueron textos enriquecidos con recursos multimediales.

Del mismo modo, el segundo grupo de estudiantes desarrolló un proyecto denominado "Bogotá a través de nuestros ojos" (Bogotá Through our Eyes). Para su realización, los estudiantes escogieron los temas que querían explorar, luego se los distribuyeron y cada grupo buscó información, fotos, videos, historias, etc. Este fue el primer intento de creación de un producto hipertextual. El resultado de la búsqueda se plasmó en el único espacio virtual diseñado por todo el grupo de estudiantes, acompañado de fotografías, varias tomadas por ellos, animaciones $\mathrm{y}$ algunos pequeños videos. Se incluyeron temas como: historia, subculturas urbanas, música, comercio, bibliotecas, parques, restaurantes, e historias contadas por habitantes de la ciudad.

\section{Wikis: Universitypedia}

El espacio denominado Universitypedia fue un wiki, que por su naturaleza facilitó la realización de proyectos colaborativos. En este espacio se crearon vínculos sobre temas generales propuestos por los diseñadores, que abarcaban la mayoría de los campos de saber de los estudiantes participantes, tales como: literatura, arte, naturaleza, geografía, historia, deportes, entre otros. Como se puede ver, estos son temas que a su vez incluyen un gran número de temáticas relacionadas entre sí. Los estudiantes universitarios escribieron varios textos sobre arte y literatura colombianos. En cuanto a arte escribieron sobre pintura, escultura y grafiti; en literatura escribieron sobre varios movimientos literarios y escritores famosos. Aunque la mayoría de los estudiantes escribieron sobre los temas anteriores, algunos lo hicieron sobre naturaleza, incluyeron temas como parques naturales y ríos. Este proyecto, al igual que los anteriores, es el resultado del trabajo en equipo.

\section{Los debates}

Otro espacio que se usó durante toda la intervención pedagógica que hizo parte de este estudio fue el espacio de debate. Cada dos semanas los estudiantes seleccionaban democráticamente un tema actual o de su interés, que por su naturaleza generara controversia, para ser discutido, y creaban una pregunta clave que orientaría la discusión. La participación siempre requirió de indagación previa sobre los temas propuestos, que consistía en leer algún artículo, ver un video o documental, etc. En ocasiones, la docente sugería fuentes en las que los estudiantes podían informarse del tema antes de participar, o cada estudiante buscaba la información autónomamente, dependiendo de su postura frente a la temática del debate. Luego, cada estudiante exponía su punto de vista en el espacio virtual y debía responder al comentario de al menos una persona participante. Las intervenciones de la mayoría de los estudiantes estuvieron acompañadas de otros recursos como imágenes, videos y enlaces a otras fuentes, elementos que sin duda le daban mayor relevancia a la opinión.

\section{Blogs personales}

En el espacio personal, semanalmente los estudiantes escribieron extensamente sobre sus gustos, 
pasiones y experiencias personales; en suma, este espacio fue diseñado y personalizado de acuerdo a los intereses de los estudiantes. Algunos escribieron sobre sus familias, amigos, vida en el colegio, lugares turísticos, sus lugares de procedencia, poemas, historias fantásticas, anécdotas, pintura, en fin, cualquier temática que les despertara su pasión por escribir. Este fue el espacio que les permitió mostrar quiénes eran ellos más allá del salón de clase, como individuos de una sociedad. Asimismo, los participantes demostraron su creatividad y conocimiento en el uso de la tecnología.

\section{Resultados y discusión}

El análisis de los datos representó un reto para los investigadores, pues se tuvo que pensar en una forma de análisis que lograra explorar, de manera apropiada y coherente, los textos escritos contenidos en los blogs, ya que hacen parte de formas de comunicación más complejas, por cuanto no se limitan al solo texto sino que se enriquecen con otros recursos como la hipermedia y el hipertexto. Esto requirió separarnos de formas tradicionales de cuantificar el lenguaje en los textos escritos y buscar un enfoque que permitiera ver el resultado de la construcción colaborativa en donde lo lingüístico, lo retórico y lo tecnológico se complementaron en el proceso de aprendizaje. Así, escogimos un método de análisis mixto (Goldman-Segall, 1998). Los autores consideran que la investigación mixta o quisitive research examina las dos caras del continuo entre las metodologías de investigación cuantitativa y cualitativa, las cuales pueden ser utilizadas para informar a la audiencia sobre lo que sucede en un campo de estudio dado. La investigación mixta es utilizada generalmente en la investigación de redes asincrónicas de aprendizaje, para describir lo que significa aprender y enseñar en redes de aprendizaje asincrónicas.

En el presente artículo reportamos una de las categorías resultantes del análisis de los datos recogidos en los blogs y las entrevistas. Esta categoría corrobora la necesidad de ofrecer un currículo innovador y de construcción conjunta con los aprendices, lo cual implica un rol propositivo y catalizador de las tensiones de aprendizaje por parte del profesor para el uso de la lengua y las TIC.

\section{Complementariedad de los saberes lingüístico y tecnológico para la comunicación}

El espacio www.ourdigitalculture.org como un ambiente virtual de aprendizaje, pretende integrar las capacidades de múltiples tecnologías para mediar la comunicación. La relación de complementariedad entre saberes lingüístico y tecnológico, se define en este estudio como el uso de las tecnologías y los recursos de la lengua como un dúo que hizo posible la participación de los estudiantes en eventos significativos de comunicación. Es decir, los estudiantes utilizaron estas dos herramientas (lengua y tecnología) como medio para comunicarse con otros interlocutores ubicados en el contexto colombiano o en internacionales. En este sentido, los saberes tecnológico y lingüístico se complementaron para facilitar y enriquecer la intención comunicativa del espacio virtual, a la vez que hicieron que los textos de los estudiantes adquirieran rasgos particulares de los textos electrónicos, evidentes en la inclusión de recursos mutimediales e hipermediales. La necesidad de uso significativo de los dos recursos (lengua y tecnología) motivó a los estudiantes a aprender cómo usarlos. Encontramos entonces que los estudiantes tuvieron unos avances realmente significativos en el uso del inglés y en el manejo operativo de algunas herramientas tecnológicas y de la Web 2.0.

El desarrollo del lenguaje escrito se hizo evidente en la capacidad descriptiva en las narraciones, historias y descripciones que los estudiantes hicieron de sí mismos, de su familia, eventos históricos y culturales de su ciudad; la capacidad argumentativa a través de la documentación de hechos cotidianos de la ciudad; la discusión crítica de algunas de las problemáticas actuales que aquejan a los jóvenes e incluso a través de la discusión crítica y argumentada de la situación educativa en Colombia. No nos centramos en reportar un análisis detallado del desarrollo lingüístico en lo que se refiere al uso de las estructuras gramaticales, vocabulario, sintaxis y otros aspectos formales de la lengua, sino más bien 
en la frecuencia con que los estudiantes usaron la lengua para construir sentido y comunicar sus ideas. Si tuviéramos que reportar la precisión en el uso de la lengua, tendríamos que aceptar que hubo muchos problemas en el uso formal de esta; sin embargo, tales problemas los consideramos propios de un aprendiz principiante de cualquier lengua extranjera.

Los estudiantes hicieron avances significativos en el uso formal del inglés, pero siempre dado gracias a la necesidad auténtica de comunicar sus ideas, gustos, habilidades, etc. Los errores siempre estuvieron presentes, pero la intención comunicativa fue exitosa: los demás participantes, en la mayoría de los casos, pudieron comprender las ideas expuestas en los textos. La ayuda de la docente y de los demás participantes fue clave para mejorar los textos. En muchos casos la participación fue espontánea, sin mucho tiempo para planear, y aun así el fin último, la comunicación, fue alcanzado. En ese esfuerzo constante por construir sentido y comunicar sus ideas, los estudiantes aprendieron, comprendieron y reforzaron la gramática de la lengua a un ritmo mucho más rápido del que se da con clases y actividades tradicionales de enseñanza, que se limitan a seguir el libro de texto. Como se mencionó al comienzo, la mayor parte del trabajo de la clase (siete horas de diez) se centraba en el libro; sin embargo, la producción escrita y oral de los estudiantes siempre mostraba que ellos usaban la lengua en formas mucho más complejas que las propuestas por el texto. Uno de los aspectos más importantes de este espacio es que motivó el uso auténtico del idioma: el hecho de escribir sobre temas de su interés los obligó a buscar los recursos lingüísticos para darle sentido a sus ideas, y en ese quehacer constante y acompañamiento de la docente, el uso de la lengua se iba perfeccionando.

El aprendizaje tecnológico se hizo evidente en el incremento de la escritura hipermedial. Encontramos que de manera cronológica, los estudiantes transitan del texto lineal complementado con fotografías, hacia la elaboración de textos básicos hipertextuales e hipermediales. Encontramos que los estudiantes, durante los primeros cuatro meses, aproximadamente, crearon textos generalmente planos y lineales y progresivamente empezaron a crear textos más complejos en su estructura, pues se elaboraron con un claro concepto hipermedial e hipertextual. La unión de texto, imagen y audio empezó a hacerse presente en un mismo escrito como recurso para dar mayor claridad a sus argumentos, hacer las descripciones más vívidas o darle más sentido a aquello que las palabras no podrían por describir sí solas. En este contexto es relevante resaltar que las frecuencias de escritura inicial muestran que hay un tiempo durante el cual se da el proceso de apropiación, tanto de las herramientas tecnológicas y digitales como de la lengua inglesa. Luego, el mismo uso intencional y significativo de cada herramienta (lengua y tecnología), la orientación del docente del curso y, muy especialmente, la colaboración entre compañeros de clase, hicieron que los estudiantes avanzaran en la comprensión y el uso de los dos recursos y lograran una comunicación cada vez más efectiva. Es por esto que, como bien lo señala el nombre de la categoría, creemos que tanto el saber lingüístico como el tecnológico se complementaron para alcanzar el fin último: la comunicación. Ambas herramientas se usaron con propósitos auténticos, en tanto el uso de los recursos tecnológicos y lingüísticos tenía una intencionalidad estrechamente relacionada con el fin último de la comunicación; es decir, dependiendo de lo que el aprendiz deseaba comunicar, buscaba los recursos tecnológicos y lingüísticos.

A continuación presentamos dos extractos de los textos que evidencian las relaciones anteriormente descritas. En el primero vemos el uso de fotografía, audio y texto.

Imagen 1. Uso de fotografía, audio y texto.

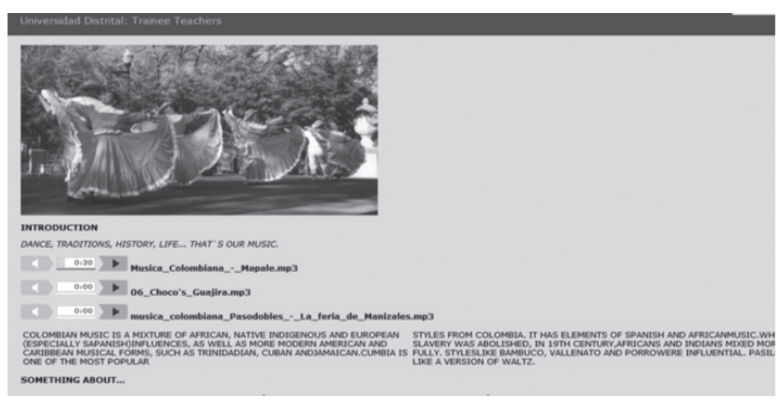


Imagen 2. Extracto de debate sobre bulimia y anorexia.

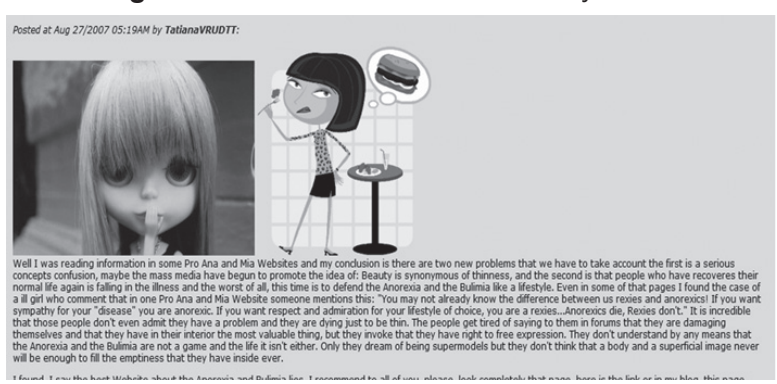

Un participante anotó: “A mí me pareció chévere del blog que no solo incluía texto, porque texto sería solamente aburrido, me pareció interesante que se pudiera incluir imágenes y animaciones y video”. (S1, entrevista, líneas 309-311, 2007)

En cuanto a la colaboración entre pares un estudiante expresó:

...uno siempre al entrar en un blog y ver que esa persona le puso algo nuevo, como que a uno le entraba la curiosidad, por decirlo así, de cómo lo hizo, de dónde lo sacó, siempre hubo una comunicación hasta extra clase para decirle oye cómo hiciste eso, cómo hiciste lo otro, no mira si quieres te presto el programa si quieres, porque a mi me sucedió, te presto el CD para el programa. Entonces siempre hubo una interacción y un aprendizaje bastante completo. (S7, entrevista, líneas 145-151, 2007)

\section{Complementariedad entre los contenidos escolares y los contenidos propios del estudiante en el aprendizaje}

La segunda relación de complementariedad en la categoría que aquí reportamos se da entre los contenidos del currículo oficial del curso y los contenidos de los estudiantes que se basan en su vida, intereses y entorno social. Estos dos contenidos se pueden apreciar en los productos plasmados en el espacio virtual. Esta relación se dio gracias a la integración que se dio entre el currículo de la universidad y el entorno sociocultural de los estudiantes. Vemos que las temáticas sociales como el valor histórico de su ciudad, la música y el teatro como riquezas culturales, las subculturas urbanas, la anorexia y la bulimia, fueron de gran interés para los estudiantes universitarios y a partir de ellas se generó un pro- pósito auténtico para usar el idioma extranjero para hablar y pensar sobre sus propios intereses, a fin de reflexionar críticamente sobre su propia realidad y dar una mirada a su propia cultura e historia.

Así, los contenidos del curso propuestos por la docente se enriquecieron con los propuestos por los estudiantes. Del mismo modo, el espacio de estudio ya no fue solo el salón de clase o el aula de informática: este se extendió a los demás territorios, el de la casa y vecindarios, museos y parques, que fueron los espacios alrededor de los cuales generaron sus narrativas y proyectos colaborativos. Los ejemplos que se incluyen a continuación ilustran explícitamente nuestra discusión:

Imagen 3. Blog Personal, S5, 2007.

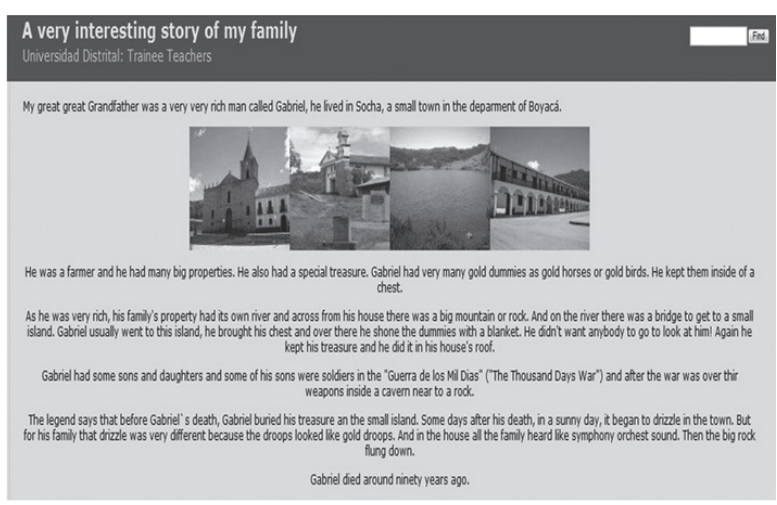

Imagen 4. Proyecto: Bogotá Through our Eyes, S1, 2007.

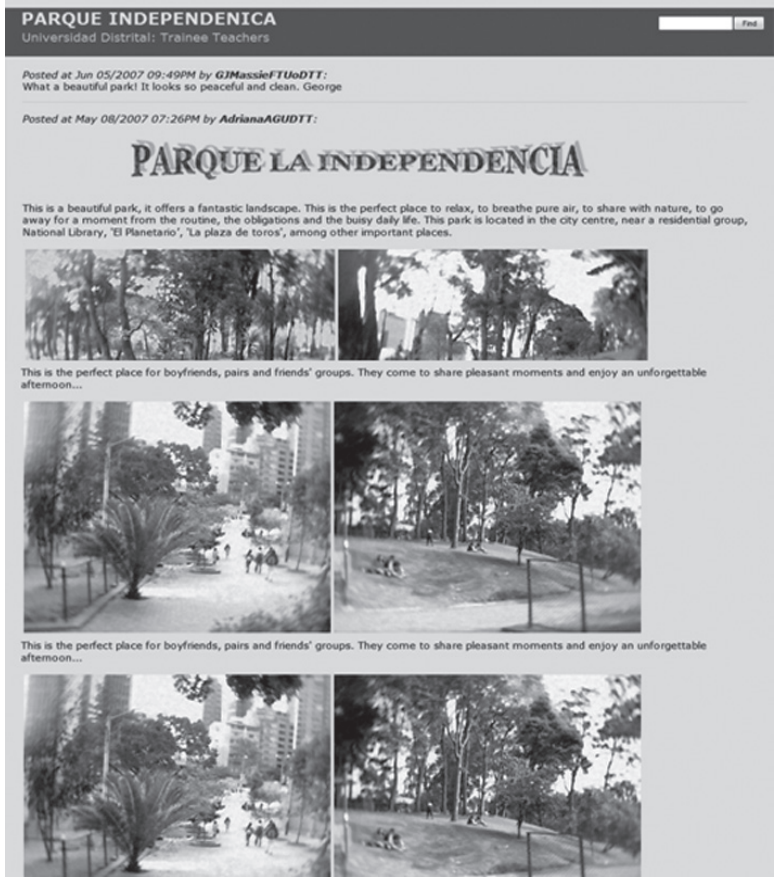


Estas nuevas circunstancias generadas por la interacción comunicativa, son un claro ejemplo de las implicaciones del currículo, que pareciera salirse de las manos y el control del docente. Frente a una tradición en la que el docente ha mantenido este control, esta es una situación de choque y de mixtura entre la condición previa y la emergente. Un currículo dinamizador, desde nuestra perspectiva, contempla una transformación del currículo prescriptivo y controlado en la acción educativa, a un currículo que permite al estudiante complementar los contenidos institucionales para comunicarse con otros, descubrir nuevas formas de aprender, narrarse a sí mismo, construir su identidad y aprender desde la indagación orientada por el docente en nuevos espacios de aprendizaje virtuales. Al respecto, Salmasi (2007) considera que "un currículo con un fuerte énfasis en lo comunicacional rescatará la investigación, más que como actividad especializada, como una actitud, una curiosidad y predisposición a reflexionar sobre temas y problemas a los cuales se aproxima por diversas vías, valiéndose de los recursos necesarios y disponibles" (p. 205). En nuestro caso, la lengua y la tecnología fueron recursos valiosos para que los estudiantes plasmaran sus propios saberes. Las entrevistas a los estudiantes que participaron en el proyecto demuestran que el proyecto trascendió las fronteras del aula de clase y les dio la opción de explorar su ciudad, literatura, arte, entorno social, entre otros.

El espacio de "Bogotá, Through our Eyes" me pareció muy interesante porque era un solo espacio pero construido por todos, entonces ahí era, solo una, es Bogotá pero había muchísimos temas que cada persona exploraba desde sus vivencias y desde sus intereses. Un espacio que yo creé era una historia de, pues cerca de mi casa, como una leyenda o un mito que sé que nadie conocía, pues porque es de allá, entonces averigüé un poco acerca de esto, un sitio que se llama el "Palo del Ahorcado" y pues conseguí una fotografía del sitio y traduje la historia. La traducción fue un poco difícil, eso me exigía mucho, ir más allá de lo que una iba viendo en la clase y ahí iba uno aprendiendo cosas del idioma, no solo de crear texto y de imágenes, sino del idioma, como que yo quería expresar muchas ideas...(S1, entrevista, líneas 296-306, 2009)

Otro estudiante expresó:

... Además de que aprendíamos mucho de lo que los otros pensaban, y son enseñanzas importantes para uno, por ejemplo cuando Tatiana expresó todo lo que hablaba de Apocalipsis, son cosas que de pronto uno no sabía, y pues es como importante saberlo, uno debe saber de todo, entonces eso me parecía muy bueno, era como una crítica pacífica, cuando hacíamos los debates, porque de pronto si tu pensabas diferente, yo pienso diferente, pero entonces...podíamos mostrar lo que sabíamos sin hacer ningún tipo de riña, sino simplemente estábamos mostrando e interactuando. (S2, entrevista, líneas 194-200, diciembre, 2007)

\section{Conclusiones}

Los resultados de la intervención pedagógica de dos semestres con los estudiantes universitarios en formación como docentes, indicaron que la pedagogía propuesta basada en la colaboración, participación y la comunicación entre estudiantes, promovió el aprendizaje del inglés como lengua extranjera y la apropiación de algunas herramientas tecnológicas en el contexto de la comunicación.

De otra parte, la formas de comunicación variadas, a través de blogs y videoconferencias, el acompañamiento de los docentes y las temáticas de trabajo propuestas en el desarrollo de proyectos colaborativos con los estudiantes universitarios, transformaron el currículo y lo hicieron más participativo. Es precisamente esa posibilidad de participar en la construcción del currículo lo que hizo que los intereses de los estudiantes estuvieran allí, que su voz hiciera parte del currículo institucional y esto, a su vez, generó el deseo de usar el idioma para expresar sus ideas, gustos, intereses y hasta sueños.

Los proyectos pedagógicos colaborativos permitieron aprovechar las habilidades linguísticas y tecnológicas de los estudiantes en cuanto a redacción, organización de ideas, creatividad en el diseño de las páginas y manejo de imágenes, para aprender sobre aspectos académicos, sociales y culturales de su interés, que enriquecieron la experiencia 
escolar e hicieron significativo el aprendizaje. Esta dinámica de aprendizaje colaborativo les mostró a los estudiantes, futuros docentes de inglés, formas de aprender a aprender y promovió su interés por explorar diferentes temáticas, sobre las que debieron indagar en grupo o recurrir a sus propias vivencias. Los proyectos pedagógicos desarrollados con los estudiantes motivaron una interacción con pares de otros países o de otras instituciones, para aprender sobre aspectos académicos, sociales y culturales de su interés que, como dijimos, enriquecieron la experiencia escolar e hicieron significativo el aprendizaje.

Los cambios en las metodologías de la enseñanza, la actitud de apertura al cambio, la gestión que se llevó a cabo a nivel institucional y las dinámicas de trabajo utilizadas por la docente con sus estudiantes, dentro y fuera del aula, representan transformaciones que impactaron positivamente el aprendizaje de los estudiantes universitarios en formacion como nuevos profesores. La participación de los estudiantes, a través de la escritura en los blogs como espacios de comunicación auténtica con otros, generó una productividad que sobrepasó las expectativas de desarrollo de la lengua, dado que los estudiantes eran estudiantes de inglés básico. Así, sus producciones muestran saberes más complejos de la lengua con respecto a los que se proponían en el currículo del curso.

Uno de los propósitos más importantes que se lograron con el trabajo fue brindarle a los futuros docentes una experiencia de aprendizaje del idioma enriquecida con la inclusión de TIC, de tal forma que se pudiera convertir en un modelo para ellos como futuros docentes. No buscábamos que hicieran una reflexión pedagógica inmediata, pues eran estudiantes de primero y segundo semestres. Sin embargo, algunos estudiantes hicieron la reflexión, tal como lo evidencia el siguiente apartado de una entrevista:

También yo vi el blog como una oportunidad de formarnos como maestros en el sentido de que nosotros, como futuros profesores, tenemos pues que estar pensando en una metodología o en unas estrategias para hacer llamativo lo que estamos enseñando, entonces pues creo que en ese blog como que nosotros jugamos dos roles, el de maestro como el de estudiante, porque nosotros debemos como maestros preparar una estrategia, que lo que ponemos en el blog sea llamativo para los otros, pero al mismo tiempo que ayude a mejorar el inglés; y somos estudiantes en el sentido en que es por nuestro propio proceso que estamos mejorando ese inglés. (S5, entrevista, líneas 134139, diciembre, 2007)

Así, al igual que Howard (2002), creemos que el incremento en la participación de los futuros docentes en este proyecto pedagógico mediado por tecnología, resultó en correspondencia con un incremento en las posibilidades para incorporar las TIC en sus futuras prácticas de enseñanza. Creemos que los educadores y los programas de formación de docentes en Colombia deben brindar experiencias de aprendizaje enriquecidas con el uso de las TIC, de manera que sea una forma de hacerlos conscientes de las implicaciones que estas tienen en el aprendizaje y la enseñanza en la escuela, y brindar modelos exitosos del uso de las TIC en la enseñanza y aprendizaje. De los anteriores resultados concluimos que este tipo de pedagogía que promueve la participación y la comunicación entre los aprendices en un ambiente virtual como el www.ourdigitalculture.org favorece el aprendizaje colaborativo, el desarrollo de competencias de escritura y comunicación en inglés como lengua extranjera y el desarrollo de competencias en TIC.

\section{Referencias}

Chan, A. \& Lee, M. (2007). We Want to be Teachers, Not Programmers: In Pursuit of Relevance and Authenticity for Initial Teacher Education Students Studying an Information Technology Subject at an Australian University. In Electronic Journal for the Integration of Technology in Education, Vol. 5, 79-97.

Clavijo, A., Quintana, A. y Quintero, L. (2011). Enseñanza de inglés y medios digitales: nuevos retos y posibilidades para la escuela. Bogotá: Universidad Distrital.

Freire, P. \& Macedo, D. (1987). Literacy. Westport, CT: Greenwood Publishing Group Inc.

Howard, J. (2002). Technology-Enhanced Project-Based Learning in Teacher Education: Addressing the Goals of Transfer. In A. Chan \& M. Lee (2007), We Want 
to be Teachers, Not Programmers: In Pursuit of Relevance and Authenticity for Initial Teacher Education Students Studying an Information Technology Subject at an Australian University (pp. 79-97).

Jacobsen, M., Clifford, P. \& Friesen, S. (2002). Preparing Teachers for Technology Integration: Creating a Culture of Inquiry in the Context of Use. In Contemporary Issues in Technology and Teacher Education [Online serial], 2(3). Retrieved July 16th, 2011, from http://www.citejournal.org/vol2/iss3/currentpractice/article2.cfm

Goldman-Segall, R. (1998). Points of Viewing Children's Thinking: A Digital Ethnographer's Journey. Mahwah, NJ: Lawrence Earlbaum Asociates.

Kalman, J. (2003). El acceso a la cultura escrita: la participación social y la apropiación de conocimientos en eventos cotidianos de lectura y escritura. En Revista Mexicana de Investigación Educativa, 8(17), 37-66.

McLoughlin, C. \& Lee, M. (2008). The Three P's of Pedagogy for the Networked Society: Personalization, Participation, and Productivity. In International Journal of Teaching and Learning in Higher Education, 20(1), 10-27.

Mishra, P. \& Koehler, M. (2006). Technological Pedagogical Content Knowledge: A Framework for Teacher Knowledge. In Teachers College Record, 108(6), 1017-1054.

Moll, L. (Ed.). (1990). Vygotsky and Education: Instructional Implications and Applications of Sociohistorical Psychology. New York, NY: Cambridge University Press.

New Media Consortium. (2005). A Global Imperative: The Report of the 21st Century Literacy Summit. Austin, TX: New Media Consortium. Retrieved March 18th, 2007, from http:www.nmc.org/pdf/ Global_Imperative.pdf.

Plan TIC. Ministerio de las Tecnologías de la Información y las Comunicaciones. (2008). Documento en línea. Recuperado el 17 de abril de 2010. Disponible en www.colombiaplantic.org.co/index.php

Short, K. (2001). The Authoring Cycle. In Colombian Applied Linguistics Journal, 22-33.

Siemens, G. (2007). Digital Natives and Immigrants: A Concept Beyond its Best Before Date (Weblog). In Connectivism Blog. Retrieved November 4th, 2007, from http://connetivism.ca/blog/2007/10/digital_naties_and_immigrants.html
Una experiencia de formación inicial de docentes de inglés para la inclusión de las TIC en la enseñanza de las lenguas
Amparo Clavijo / Luz Mary Quintero

Vygotsky, L. (1978). Mind in Society. Cambridge: MA. Harvard University Press.

Wells, G. (1999). Dialogic Inquiry: Towards a Sociocultural Practice and Theory of Education. New York: Cambridge University Press.

Universidad de Dundee. (Escocia). Project Forum. Retrieved from www.ourdigitalculture.org.

Zhao, Y. \& Bryant, F. (2007). Can Teacher Technology Integration Training Alone Lead to High Levels of Technology Integration? A Qualitative Look at Teachers' Technology Integration After State Mandated Technology Training. In Electronic Journal for the Integration of Technology in Education, Vol. 5, 54-70. 